\title{
Análisis de la dinámica relacional generada por las websites de Internet en el desarrollo de los turismos marginales ${ }^{1}$
}

\author{
José $\mathbf{M}^{\mathrm{a}}$ Prat ${ }^{\mathrm{i}}$ \\ Univesidad Autónoma de Barcelona (España)
}

\begin{abstract}
Resumen: El conocimiento de los actores turísticos del territorio, y de sus relaciones, es un elemento básico para la gestión activa de cualquier destino turístico. Como que, gracias a las nuevas tecnologías de la información y a la popularización del uso de Internet, se han creado una gran cantidad de relaciones sociales gracias a las websites, interrelacionadas, que han permitido una rápida y amplia difusión promocional de aquellos destinos que ofrecen unas alternativas turísticas más marginales, como respuesta a los nuevos planteamientos post-fordistas del turismo, generándose una dinámica relacional que se ha beneficiado del rápido desarrollo de las TIC's. En este trabajo se han analizado dichas relaciones entre websites, en el caso del desarrollo de dos turismos marginales, el naturista y el swinger, en Cap d'Agde, en el sur de Francia, comprobándose la existencia de una relación directa entre la dinámica relacional del territorio y el grado de desarrollo de los turismos marginales.
\end{abstract}

Palabras clave: Turismo marginal; Turismo swinger; Turismo naturismo; Websites; Análisis de redes; Relaciones sociales.

Title: Analysis of relational dynamics generated by Internet websites in the development of marginal tourism

\begin{abstract}
Knowledge of tourism stakeholders on the territory, and its relations, is a basic element for the active management of any tourist destination. The new information technologies and the popularization of Internet, have created a lot of social relationships among the websites linkeded that have enabled a rapid and widespread promotion of marginal tourist destinations, in response to new approaches to post-fordist tourism, generating a dynamic relationship, benefited of the rapid development of TIC's. In this paper we have examined these relationships among websites, in the case of development of two marginal tourisms in the south of France, in Cap d'Agde, where both tourisms, naturist and swinger, proving the existence of direct relationship between websites in order to confirm the relational dynamics on the territory and the degree of development of marginal tourisms.
\end{abstract}

Keywords: Marginal tourism; Swinger tourism; Naturist tourism; Websites; Network analysis; Social relationships.

i Departamento de Geografía. Universidad Autónoma de Barcelona. E-mail: josepmaria.prat@campus.uab.es 


\section{Introducción}

En el actual contexto post-fordista del turismo, caracterizado por una mayor experiencia de los turistas, más disponibilidad de tiempo libre, hábitos consumistas y un poder adquisitivo medio superior, nos encontramos en un mercado con cambios constantes y demandas en permanente modificación (Priestley y Llurdés, 2007), que tienen que ver con actitudes y no simplemente con comportamientos, de modo que dichas actitudes marcan el camino hacia el nuevo modelo de consumo de los bienes y servicios turísticos (Oliver y Alloza, 2009).

Por ello, en estas últimas décadas, la explosión del turismo ha servido para que los destinos, con una buena promoción, puedan ser utilizados por amplios grupos de turistas, creándose ofertas específicas y personalizadas para cada colectivo concreto que desea dar sentido a su tiempo de ocio. Los turismos marginales son algunas de las estrategias utilizadas para la reactivación de los destinos turísticos con el fin de evitar su declive, siguiendo el ciclo de vida propuesto por Butler (1980) ${ }^{2}$.

Por otra parte, en estos últimos años, gracias a los avances de las tecnologías de la información y las comunicaciones (las denominadas TIC's), se ha consolidado la sociedad de la información, constituyéndose un nuevo sistema tecnológico, económico y social, en el que la generación, el proceso y la transmisión de la información y del conocimiento son fuentes fundamentales de productividad y poder, dando lugar a una situación de cambio global en un mundo en red (Pérez et al. 2003).

De este modo, las TIC's se han convertido en una herramienta cada vez más utilizada por los viajeros a la hora de planificar viajes y elegir sus destinos turísticos, ya que en los portales de Internet (las "websites") es posible encontrar mucha información referente a las características de las ofertas presentes en los destinos; así como las opiniones de otros turistas ${ }^{3}$.

Ante esta situación, creemos que el desarrollo del turismo marginal depende cada vez más de la capacidad del destino para crear y mantener redes de colaboración ${ }^{4}$ entre los diferentes agentes, privados y públicos, presentes en el territorio, vinculadas económicamente, geográficamente, socialmente y culturalmente (Ávila y Barrado, 2005), de manera que el conocimiento de los actores y de las relaciones que se generan en la red resultan fundamentales para el desarrollo turístico del destino.

En este punto hay que indicar que la mayoría de autores que trabajan el tema de las redes están de acuerdo en definir una red social como un conjunto de relaciones que no implican en sí mismas ni una conciencia organizadora, ni un sentimiento de pertenencia, ni una frontera (Grossetti, 2009).

En consecuencia, las relaciones que se generan a través de las redes sociales creadas gracias a los "links" presentes en los portales de Internet, no siendo formales, generan un entramado de informaciones y conocimientos que ayudan mucho a la promoción globalizadora del turis- mo en un determinado destino.

Dos de estas ofertas de turismo marginal son el turismo naturista y el turismo swinger. El primero, el turismo naturista, tiene como objetivo vivir plenamente el naturismo como concepto de vida en armonía con la naturaleza, con igualdad entre la gente y sin discriminación de género; siendo norma la práctica del nudismo colectivo en todos los actos de la vida cotidiana, favoreciendo la tolerancia y el respeto por uno mismo, por los demás y por el medioambiente (Norrild, 2007).

El segundo, el turismo swinger, se fundamenta en el intercambio temporal y consentido de los miembros de una pareja estable ${ }^{5}$ con fines básicamente sexuales (Bergstrand y Blevins, 2000).

Ambas ofertas turísticas conjugan naturaleza, sol y playa, pero están dirigidas a diferentes colectivos y generan relaciones desiguales sobre el territorio (Allen et al., 1998; Paasi, 2002; Suvantola, 2002).

En este contexto, siguiendo la línea argumental de Merinero y Pulido (2009), que indican que existe una relación directa entre la dinámica relacional que se produce entre los agentes implicados en el desarrollo turístico de un destino y el nivel que alcanza este desarrollo, de manera que a mayor intensidad de dichas relaciones mayor es el desarrollo turístico del territorio, en el presente trabajo se han analizado las características de las relaciones establecidas entre los "links" existentes en las distintas "websites" de Internet que intervienen en la divulgación de la información sobre dos turismos marginales, el naturista y el swinger, en una importante población turística de la costa mediterránea francesa, el Cap d'Agde y su barrio naturista.

Por ello, en este artículo se proponen demostrar las tres hipótesis siguientes:

$\left.1^{a}\right)$ Las relaciones sociales generadas a través de los "links" existentes en las "websites" de Internet favorecen el desarrollo turístico de los destinos donde existe un cierto turismo marginal

$2^{a}$ ) La dinámica relacional que generan en un determinado destino las "websites" de las comunidades virtuales relacionadas con el turismo marginal allí presente, es fundamental en el desarrollo de este turismo en el destino

$3^{\circ}$ ) Los potenciales usuarios de estos turismos marginales perciben la utilidad de las opiniones, comentarios y experiencias que otros turistas presentan en las "websites" de dichas comunidades virtuales y, en general, se suelen seguir sus recomendaciones

Finalmente, la pertinencia de este trabajo se fundamente en el hecho de que los análisis relacionales han sido objeto de poco interés en la literatura académica hasta hace pocos años, ya que la mayoría de los estudios se han focalizado en la perspectiva del actor y no tanto en las relaciones que se producen entre dichos actores (Merinero y Pulido, 2009). 


\section{El turismo marginal en Cap d'Agde}

Cap d'Agde es una población turística situada en el sur de Francia, perteneciente a la comuna de $\mathrm{Agde}^{6}$, en el departamento de Hérault (Languedoc-Rousillon), situada en el mar Mediterráneo, que dispone de un barrio naturista, cerrado y controlado, desarrollado a partir de los años 70's con la ayuda de las administraciones públicas francesas, con el objetivo de potenciar la oferta turística naturista de la zona, buscando captar los flujos de turistas estivales marginales que, desde el norte y centro de Europa, iban hacia España en busca de sol y playa, pero cuyo régimen político, aún con importantes residuos franquistas, rechazaba frontalmente la práctica pública del nudismo (Prat, 2010; Harp, 2011).

A principios de los 80 's, el perímetro del barrio naturista de Cap d'Agde ya alcanzó su dimensión actual, incorporando urbanizaciones de chalets y apartamentos, un hotel, un camping, varios restaurantes y bares, comercios y servicios de todo tipo, piscinas, zonas deportivas y de ocio, una playa de $2 \mathrm{~km}$ y un puerto deportivo con 300 plazas de embarcadero.

Sin embargo, a principios de los 90's, aprovechando las instalaciones y los servicios existentes en el barrio naturista, allí también se inició el turismo swinger, al inaugurarse en el interior del recinto el complejo lúdico-comercial de Villa Romana, con varios clubes de intercambios de parejas, y convirtiéndose, con el tiempo, en el primer centro swinger de Europa ${ }^{7}$, aunque en estos últimos años han empezado a aflorar conflictos entre ambos colectivos de turistas, debido al uso de los espacios públicos del barrio ${ }^{8}$.

Así pues, el barrio naturista de Cap d'Agde es una pequeña ciudad, cerrada y controlada, con una discreta red de vigilantes privados y policía local, bajo gestión municipal, cuya población estival, nudista, ejerce como tal no solo en la playa sino en todos los espacios del barrio.

\section{a) Metodología aplicada}

El planteamiento metodológico de esta investigación parte del reconocimiento de la importancia que tienen las relaciones entre los actores en un destino turístico, en este caso el barrio naturista de Cap d'Agde.

Para comprobar las hipótesis expuestas, se han obtenido datos desde tres fuentes distintas:

a) la prospección personal de "links" en los portales de Internet relacionados con el barrio naturista de Cap d'Agde

b) aprovechamiento de algunos de los resultados obtenido a través de una encuesta realizada mediante cuestionario en Cap d'Agde ${ }^{9}$

c) datos cuantitativos extraídos del Instituto Nacional francés de Estadística (INSEE)

Así pues, en el primer caso (la prospección de "websites") se trataba de identificar los principales actores que intervienen en la promoción del barrio naturista de Cap d'Agde, a partir de los "links" entre los portales existentes en Internet (tanto de organismos públicos como de entidades y asociaciones privadas), con el objetivo de analizar las relaciones sociales establecidas entre ellos.

Por ello, tal como se puede observar en la tabla siguiente (fig.1), se han definido nueve grupos de actores relacionados con estos dos tipos de turismo marginal de Cap d'Agde.

A continuación, una vez definidos estos 9 grupos, se ha procedido a identificar los actores más relevantes en cada uno de estos grupos, para lo cual se ha utilizado una técnica similar a la denominada "bola de nieve" ("snowballing”) (Granovetter, 1976; Erickson et al., 1981, 1983; Frank y Snidjers, 1994; Molina, 2001), de manera que mediante fuentes indirectas de información se ha constituido una lista inicial de 7 actores pertenecientes a algunos de los colectivos previamente identificados; que se ha ido ampliando en dos oleadas sucesivas.

Las "websites" seleccionadas en el primer nivel de búsqueda fueron las siguientes:

- Las de las dos agencias inmobiliarias del barrio naturista (AGN y Agencia Oltra)

- Las del hotel nudista (Eve) y la del camping del barrio naturista (Camping Oltra)

- La de la oficina de turismo de Cap d'Agde

- La municipal de Cap d'Agde

- La del barrio naturista de Cap d'Agde

Seguidamente, a partir estas 7 "websites" iniciales, se formó una segunda lista con las websites de los nuevos actores que iban aparecido en cada uno de estos portales, y que estaban relacionados de alguna manera con estos tipos de turismo marginal. Esta segunda lista se formó con las "websites" de 28 nuevos actores que también fueron encuadrados en el correspondiente grupo de la fig.1.

Finalmente, a partir de estas 28 "websites" de la segunda lista, se confeccionó una tercera lista, apareciendo otros 68 nuevos actores diferentes, que también fueron encuadrados en su correspondiente grupo inicial (fig.1).

En total, se obtuvieron 103 actores distintos, todos

\begin{tabular}{|l|l|}
\hline \multicolumn{4}{|c|}{ descripción } \\
\hline Las oficinas de turismo de la zona \\
\hline $\begin{array}{l}\text { Los distintos organismos de las administraciones públicas de la zona } \\
\text { (ayuntamientos, comunas, departamentos, regiones) }\end{array}$ \\
\hline Los clubs swingers de la zona \\
\hline Las asociaciones naturistas \\
\hline $\begin{array}{l}\text { Los hoteles y restaurantes directamente relacionados con estos turismos } \\
\text { marginales en un radio de 10 km del barrio naturista }\end{array}$ \\
\hline Las principales agencias de viajes e inmobiliarias de la zona \\
\hline Los portales privados de promoción turística de Cap d'Agde \\
\hline Los portales de promoción de las asociaciones empresariales \\
\hline Los portales de comunidades de swingers \\
\hline
\end{tabular}

Figura 1. Lista de los grupos de actores preseleccionados para el análisis de la red (fuente: elaboración propia) 


\begin{tabular}{|l|c|c|c|}
\hline & $\begin{array}{c}n^{\circ} \text { actores } \\
\text { diferentes } \\
\text { encontrados }\end{array}$ & $\begin{array}{c}n^{\circ} \text { total de actores } \\
\text { diferentes } \\
\text { acumulados }\end{array}$ & en \% \\
\hline Primer nivel de búsqueda & 7 & 7 & $6,8 \%$ \\
\hline segundo nivel de búsqueda & 28 & 35 & $33,8 \%$ \\
\hline tercer nivel de búsqueda & 68 & 103 & $59,4 \%$ \\
\hline$n^{\circ}$ total actores diferentes & & 103 & $100 \%$ \\
\hline
\end{tabular}

Figura 2. Cantidad de actores seleccionados para el análisis de la red, según el nivel de búsqueda (fuente: elaboración propia)

\begin{tabular}{|c|l|c|c|}
\hline nodos & \multicolumn{1}{|c|}{ descripción } & $\begin{array}{c}\text { websites } \\
\text { encontradas }\end{array}$ & en \% \\
\hline 1 al 5 & Websites de las oficinas de turismo & 5 & $4,86 \%$ \\
\hline 6 al 9 & $\begin{array}{l}\text { Websites de las administraciones públicas } \\
\text { (ayuntamientos, comunas, departamentos, regiones) }\end{array}$ & 4 & $3,89 \%$ \\
\hline 10 al 28 & Websites de los clubs swingers & 19 & $18,45 \%$ \\
\hline 29 al 34 & Websites de las asociaciones naturistas & 6 & $5,82 \%$ \\
\hline 35 al 41 & Websites de los hoteles y restaurantes & 7 & $6,80 \%$ \\
\hline 42 al 47 & Websites de las agencias de viajes e inmobiliarias & 6 & $5,82 \%$ \\
\hline 48 al 59 & Websites privadas de promoción del territorio & 12 & $11,64 \%$ \\
\hline 60 al 66 & Websites de las asociaciones empresariales & 7 & $6,80 \%$ \\
\hline 67 al 103 & Websites de las comunidades swingers & 37 & $35,92 \%$ \\
\hline
\end{tabular}

Figura 3. Codificación de los actores encontrados para el análisis de la red (fuente: elaboración propia)

$\begin{array}{llllll}\triangle & \text { 1al } 5 & \mathbb{1} & 29 \text { al } 34 & \square & 48 \text { al } 59 \\ \nabla & 6 \text { al } 9 & \triangleright & 35 \text { al } 41 & \square & 60 \text { al } 66 \\ & 10 \text { al } 28 & \nabla & 42 \text { al } 47 & \square & 67 \text { al } 103\end{array}$

Figura 4. Leyenda de los diferentes grupos de actores de la red (fuente: elaboración propia)

ellos relacionados de alguna manera con el barrio naturista y el turismo marginal de Cap d'Agde, tal como se detalla en la tabla siguiente (fig.2).

Una vez encuadrados los 103 actores encontrados, se les asignó un nodo de la red a cada uno de ellos y se procedió a su codificación numérica, tal como se especifica en la tabla siguiente (fig.3).

Como se aprecia en la tabla anterior (fig.3), casi el 36\% de las "websites" encontradas correspondían a portales de comunidades swingers, la mayoría franceses, mientras que, entre las restantes, destacaban los portales de los clubs swingers de la zona de Cap d'Agde (el 18,45\%) y de las agencias de promoción turística del territorio (el $11,64 \%)$.

El siguiente paso fue tabular las relaciones existentes entre estos 103 actores mediante una matriz binaria ${ }^{10} \mathrm{y}$ rectangular de modo-1 (es decir, con la misma serie de actores en las filas y columnas), simétrica y con los vínculos no orientados, visualizándose dicha matriz mediante un sociograma obtenido con el programa NetDraw (Borgatti, 2002), manteniendo la diferencia visual entre los 9 grupos de actores, aplicando diferentes formas tipológicas para cada grupo y distintos colores de relleno: las entidades más directamente relacionadas con el turismo swinger (en color negro), el turismo naturista (en color gris oscuro) y el resto (en color gris claro), tal como se presenta en la leyenda siguiente (fig.4).

A continuación, con el programa Ucinet 6 (Borgatti et al, 2002) se analizaron las relaciones sociales existentes entre los distintos actores de la red, centrándonos en las medidas de cohesión, es decir, la presencia de lazos a través de la centralidad (Sanz, 2003), frente a la posibilidad de buscar las posiciones (la llamada "equivalencia estructural"), ya que los resultados obtenidos son similares con ambas aproximaciones (Molina, 2001).

Después, a partir de la matriz binaria inicial, con Ucinet 6 y Netdraw, se crearon diferentes matrices relacionales para comprobar las hipótesis aquí planteadas.

En el segundo caso (datos de la encuesta), los datos utilizados en este artículo forman parte de unos resultados más amplios obtenidos de una encuesta aleatoria, realizada mediante cuestionario, a nivel personal e individual $^{11}$, aunque hay que indicar que somos conscientes de que estas entrevistas no pueden considerarse como una información empírica (García Muñoz, 1991) y que no están exentas de problemas de subjetividad en el momento 
de realizar el análisis; pero, aún así, creemos que son un medio para obtener una visión de la percepción que tienen los informantes, turistas y la comunidad local de Cap d'Agde sobre el barrio naturista y los turismos marginales que allí se desarrollan.

\section{b) Comprobación de las hipótesis planteadas}

Hipótesis 1: Las relaciones sociales generadas a través de los "links" existentes en las "websites" de Internet favorecen el desarrollo turístico de los destinos donde existe un cierto turismo marginal

La evolución de la población turística estival en el barrio naturista de Cap d'Agde, a lo largo del período 19752010, ha sido tal como se presenta en el gráfico siguiente (fig.5); donde, además, se compara también con la producida, para el mismo período, en la población turística estival de todo el núcleo urbano de Cap d'Agde y también con la de la población oficialmente censada en la comuna de Agde (a la que pertenece dicha población)

Como se aprecia en el gráfico anterior (fig.5), la evolución de la población estival, tanto en el barrio naturista como en todo el núcleo urbano Cap d'Agde, ha ido creciendo constantemente a lo largo de todo el período ${ }^{12}$; resultando una población actual estival en el barrio naturista que se encuentra muy cercana a las 50.000 personas, y la población estival en todo el núcleo de Cap d'Agde de 225.000 personas.

Adermás, el volumen turístico del núcleo urbano de Cap d'Agde (turismo familiar de sol y playa) es muy superior al del barrio naturista ${ }^{13}$; lo mismo que la población permanente censada en toda la comuna de Agde. Así, en el período 1975-2010 se ha pasado de una población estival de casi 120.000 personas a otra de 225.000, es decir duplicándose la población, mientras que, para el mismo período, el turismo estival en el barrio naturista ha pasa- do de 25.000 a 50.0000 personas.

Análogamente, la población permanente de la comuna de Agde ha pasado de 11.605 (en el año 1975) a 17.583 (en el 1990), 20.1003 (en el 2000) y superar los 25.000 en la actualidad (INSEE, 2011).

Paralelamente, en Francia se ha producido un aumento notable de la cantidad de internautas desde el año 2000 y de miembros de redes sociales (fig.6), desde que en el año 1994 empezó a utilizarse Internet de manera doméstica en el país ${ }^{14}$.

Como se observa en el gráfico anterior (fig.6), en Francia, aunque el uso doméstico de Internet se inició en el año 1994, no se ha producido un crecimiento sostenido en el uso de Internet hasta el inicio de la década del 2000, pasándose de 8.500.000 internautas en el año 2000 a los

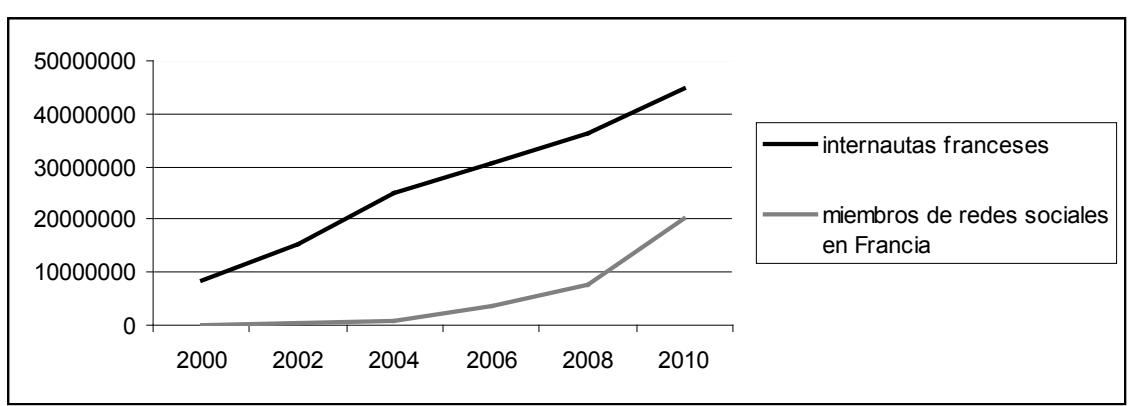

Figura 6. Evolución de la cantidad de internautas franceses y de miembros de redes sociales (2000-2010) (elaboración propia a partir de los datos extraídos de INTERNET WORLD STATS, 2010)
44.625.300 del año 2010; mientras que el despegue de los miembros de redes sociales no se ha producido hasta el año 2009, en que se han superado los 20 millones de miembros (INTERNET WORLD STATS, 2010). Además, el $92 \%$ de los internautas franceses se conectan a Internet prácticamente todos los días, y el $64 \%$ utilizan habitualmente las "websites" para buscar información sobre algún tema concreto (al respecto, el $50 \%$ de toda la población francesa declara que reserva sus vacaciones estivales a través de Internet) (Lacoste, 2010).

En consecuencia, tal como puede observarse en el gráfico siguiente (fig.7), el porcentaje de internautas respecto a la población total francesa, ha tenido una evolución

creciente a lo largo de la década, de manera que ha pasado de ser el $14,4 \%$ en el año 2000 al $68,9 \%$ en el año 2010 (INTERNET WORLD STATS, 2010).

Por otra parte, a partir de los resultados de la encuesta que hacían referencia a la manera como los turistas habían tenido conocimiento de la existencia del barrio naturista de Cap d'Agde, se ha obtenido el gráfico siguiente (fig.8), donde apreciar que el $44 \%$ de las respuestas afirmaron que su cono- 
cimiento provenía de la información adquirida a través de los portales de Internet, mientras que el 11\% dijo que lo hizo a través de revistas especializadas, el $33 \%$ por recomendación de amigos y el11\% restante no se definió al respecto. (con 50), seguida por las "websites" de los actores 43 (la agencia Inmobiliaria AGN), con 35, y el 10 (el club swinger Le Glamour), con 22; siendo la media de la red de 7,495 lazos (7,348 ya normalizada) y con una desviación estándar de 6,008 (5,89 ya normalizada).

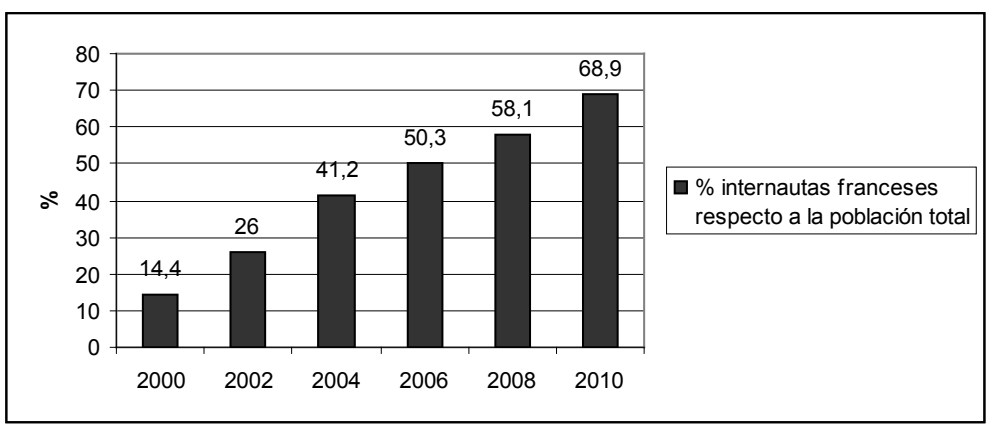

Figura 7. Evolución porcentual de la población internauta respecto a la población total francesa (2000-2010) (fuente: Prat , 2010; a partir de datos extraídos del INSEE, 2010)

Como vemos en el gráfico anterior (fig.8), el medio de mayor prescripción del barrio naturista de Cap d'Agde son los portales de Internet, sustituyendo a las revistas especializadas (naturistas y swingers), que hasta la irrupción masiva de Internet eran el medio más utilizado como prescriptor de los turismos marginales en este destino.

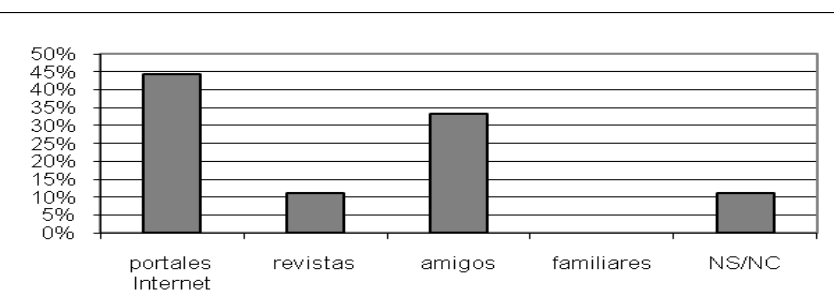

Figura 8. Medio de conocimiento inicial de la existencia del barrio naturista de Cap d'Agde (fuente: elaboración propia partir de la encuesta)

Una diferencia entre ambos tipos de turismo marginal de baja densidad (Brenner y Fricke, 2007; Cooper, 2010), es que la promoción del destino en el caso del turismo naturista se realiza fundamentalmente a través de las relaciones personales y las asociaciones naturistas, mientras que en el caso del turismo swinger, además de las relaciones personales, predominan los anuncios en revistas, portales de Internet de comunidades swingers, clubs de intercambios y agencias de viaje especializadas.

A continuación, a partir de la matriz de relaciones binarias existentes entre los 103 actores seleccionados, se realizaron los análisis de las redes sociales (con Ucinet 6); obteniéndose los siguientes resultados:

(1) Rango ("degree"): nos informa sobre el número de lazos directos de un actor, sin normalizar ("degree") y normalizados ("nrmdegree"). En nuestro caso, la red tenía una centralización del 42,50\%, con 14 actores con un rango mayor (fig.9), donde vemos que la "website" del actor 2 (la oficina de turismo de Cap d'Agde) es la que tenía mayor número de lazos directos en Internet
Otras "websites" con más de 10 lazos, aunque a niveles muy inferiores a las tres anteriores, eran la de la agencia de turismo del departamento (actor 4), la de la comuna de Agde (actor 8), las de dos de las agencias de viajes de Cap d'Agde (actores 45 y 46), la del hotel nudista Eve (actor 35) y la del restaurante de Villa Romana (actor 36), ambos en el barrio naturista, además de dos "websites" de promoción del territorio (actores 48 y 57) y tres de portales franceses de comunidades swingers (actores 67, 68 y 103).

(2) Cercanía ("closeness"): nos indica el grado de cercanía de un nodo con el resto de nodos de la red. En nuestro caso, el análisis nos

\begin{tabular}{rrrr} 
& Degree & NrmDegree & Share \\
\hline 2 & 50.000 & 49.020 & 0.065 \\
43 & 35.000 & 34.314 & 0.045 \\
10 & 22.000 & 21.569 & 0.028 \\
4 & 14.000 & 13.725 & 0.018 \\
48 & 14.000 & 13.725 & 0.018 \\
46 & 12.000 & 11.765 & 0.016 \\
103 & 12.000 & 11.765 & 0.016 \\
68 & 12.000 & 11.765 & 0.016 \\
36 & 12.000 & 11.765 & 0.016 \\
57 & 11.000 & 10.784 & 0.014 \\
67 & 11.000 & 10.784 & 0.014 \\
8 & 11.000 & 10.784 & 0.014 \\
45 & 11.000 & 10.784 & 0.014 \\
35 & 11.000 & 10.784 & 0.014
\end{tabular}

Figura 9. Lista con los catorce actores con mayor rango en la red fuente: elaboración propia con Ucinet 6

dió un grado de centralización de la red del 41,45\%, siendo los actores con una mayor cercanía también los tres de mayor rango, es decir el 2 (la oficina de turismo de Agde), el 43 (la agencia inmobiliaria AGN) y el 22 (el club swinger l'Extasia), con una media de la red del 41,774 ; mientras que el índice de lejanía ("farness") era de 248,874, con una desviación estándar de 37,155 (para la cercanía) y de 5,499 (para la lejanía). Las entidades directamente relacionadas con estos tres actores también tenían un alto índice de cercanía, de modo que, aunque estaban poco conectadas con el resto, sólo por el hecho de estar conectadas con alguna de las tres de mayor rango, ya tenían cierta influencia en el conjunto de las relaciones establecidas.

(3) Grado de intermediación ("betweeness"): nos muestra los caminos más cortos entre dos nodos. En este caso, los actores más imprescindibles (con un índice superior a 250) eran el 2 (la oficina de turismo de Agde), el 43 (la agencia inmobiliaria AGN), el 10 (el club swinger Le Glamour), el 48 (un portal de promoción de Cap d'Agde) y el 58 (una "website" de promoción del territorio), con un índice de centralización de la red del $27,85 \%$, una media de 73,437 y una desviación estándar de 177,308 . 
(4) Indice de poder de Bonacich: tiene en cuenta no sólo las relaciones de "ego" sino también las de su vecindario (Molina, 2001). En nuestro caso, los tres actores más poderosos continuaban siendo los de mayor rango e intermediación, es decir, el 2 (la oficina de turismo de Agde), con un índice normalizado de 52,827, el 10 (el club swinger Le Glamour), con 23,244 y el 43 (la agencia inmobiliaria AGN) con 36,979.

(5) Densidad: nos indica la relación existente entre el número de lazos existentes y posibles. En nuestro caso, se detectaron 772 lazos entre los 103 actores de la red, por lo que, al ser una matriz simétrica, la densidad era de $0,07^{15}$, es decir, solamente existían un $7 \%$ de lazos sobre los posibles.

(6) Agrupaciones ("Cliques"): nos permite conocer los diferentes grupos a los que pertenece un actor. En este caso, si determinamos que el número mínimo de actores para identificar un grupo es de 3, se detectó la existencia de 130 agrupaciones directas, donde todos los nodos estaban conectados entre sí, de los cuales únicamente 12 (el 9,2\%) estaban formados por cuatro o más actores, siendo los actores con mayor presencia el 10 (el club Le Glamour), el 2 (la oficina de turismo de Agde), el 43 (la agencia inmobiliaria AGN), el 48 (un portal de promoción de Cap d'Agde), el 60 (una de las asociaciones empresariales francesas) y tres de los portales de comunidades swinger (el 68, el 71 y el 103). Si tenemos en cuenta, no solamente los lazos directos, sino también los que conectan con otros nodos a distancia 2 (agrupaciones "N-cliques"), entonces se detectaron 3.826 grupos, siendo los actores con mayor pre- sencia el club Le Glamour (el actor $\left.n^{\circ} 10\right)$, la oficina de turismo de Agde (actor $n^{\circ}$ 2), la agencia inmobiliaria AGN actor $\left.n^{\circ} 43\right)$ y dos portales de comunidades swingers (actores $\mathrm{n}^{\circ} 101$ y 103). Además, había 35 grupos de actores que formaban parte del mismo grupo pero que no necesariamente conocían a todos los miembros que lo formaban (agrupaciones "N-clanes"). Otra medida de agrupaciones analizada fue el "K-plex", que se basa en que un actor puede ser miembro de un "clique" siempre y cuando tenga vínculos directos con todos miembros del grupo excepto K (Molina, 2001), de manera que K-plex muestra información sobre círculos sociales superpuestos. En este caso, si $K=2$ (un actor será miembro de un "clique" si conoce a todos menos dos actores del mismo), el resultado obtenido fue de 2.749 agrupaciones del tipo K-plex, destacando los actores 2 (la oficina de turismo de cap d'Agde), 10 (el club Le Glamour), el 43 (la agencia inmobiliaria AGN), un portal de promoción de Cap d'Agde (el 48) y una de las asociaciones empresariales (el 66).

A continuación, una vez analizada la cohesión de la red se procedió a su visualización con Netdraw, resultando el sociograma siguiente (fig.10

En el sociograma anterior (fig.10), visualmente se puede apreciar que nos encontramos ante una red muy compacta, con gran cantidad de interrelaciones entre los distintos actores ("nodos"), algunos de los cuales tienen muchas conexiones con los restantes, es decir, con una gran centralidad, tal como ya se ha puesto de manifiesto numéricamente en el análisis anterior, de manera que en estos tipos de turismo se utilizan frecuentemente las "we-

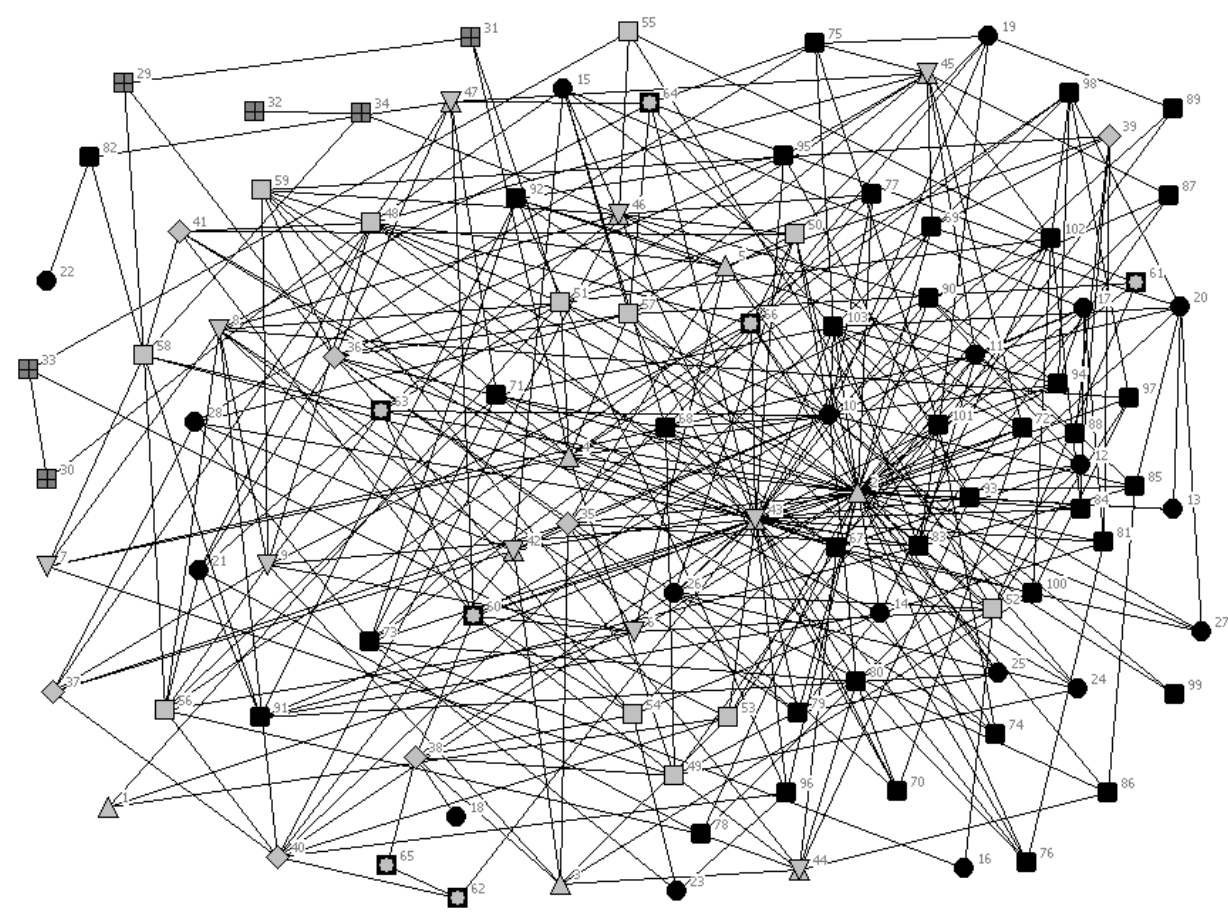

Figura 10. Red de relaciones entre todos los actores involucrados con el turismo marginal en Cap d'Agde (fuente: elaboración propia con Netdraw) 
bsites" relacionadas con el barrio naturista de Cap d'Agde para crear extensas redes sociales, donde se produce un rápido intercambio de información y opiniones (Townsend, 2002).

Así pues, las relaciones sociales generadas a través de los portales de Internet, en el caso de Cap d'Agde, favorecen el desarrollo turístico del barrio naturista, sede del turismo marginal de la región; destacando la influencia de las relaciones generadas por el club swinger Le Glamour, la oficina de turismo de Agde, la agencia inmobiliaria AGN (que es una de las dos agencias que gestiona el mercado inmobiliario del barrio naturista de Cap d'Agde) y el Patronato de Promoción Turística de Cap d'Agde.

Hipótesis 2: La dinámica relacional que generan en un determinado destino las "websites" de las comunidades virtuales relacionadas con el turismo marginal alli presente, es fundamental en el desarrollo de este turismo en el destino

Si analizamos por separado los dos tipos de turismo marginal presentes en el barrio naturista de Cap d'Agde (el naturista y el swinger) y en cada caso solamente tenemos en cuenta los actores más directamente relacionados con cada uno de ellos, obtenemos los dos sociogramas siguientes (fig.11 y 12).

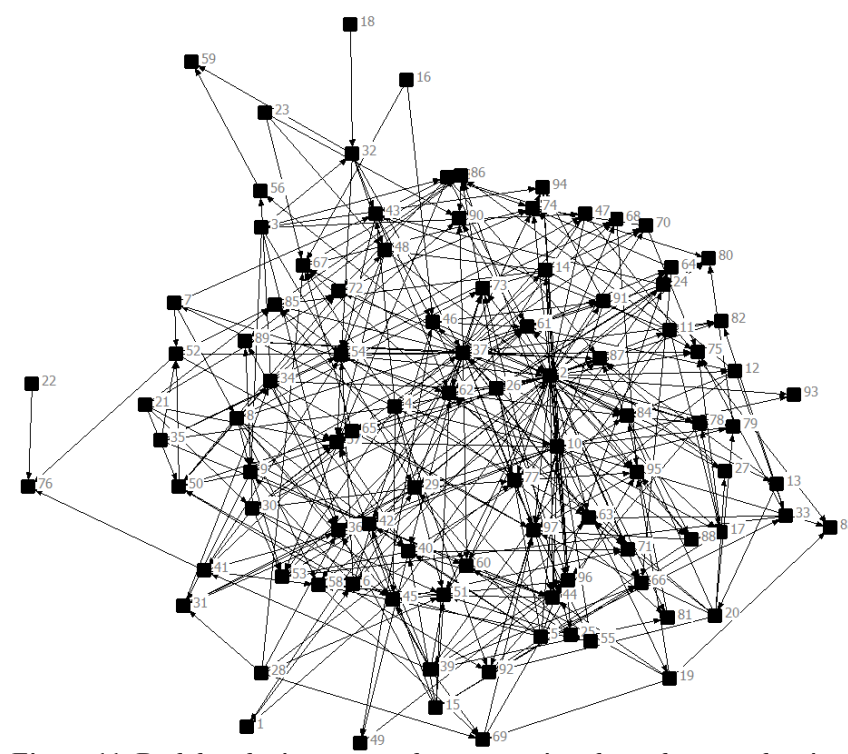

Figura 11. Red de relaciones entre los actores involucrados con el turismo swinger en Cap d'Agde (fuente: elaboración propia con Netdraw)

Como se aprecia visualmente en el sociograma anterior (fig.11), además de la menor cantidad de nodos (hay 97), se mantiene una considerable compactación de la red; destacando la conectividad de los portales de los principales clubs de intercambio, especialmente "Le Glamour" y "L'Extasia".

Análogamente, en el caso de la red de relaciones del turismo naturista (fig.12), hay muchas menos relaciones ("links") generadas por los portales de Internet relacionados de alguna manera con este tipo de turismo, debido a la existencia de menos nodos (hay 47), y con una compactación de red menor que en el caso del turismo swinger.

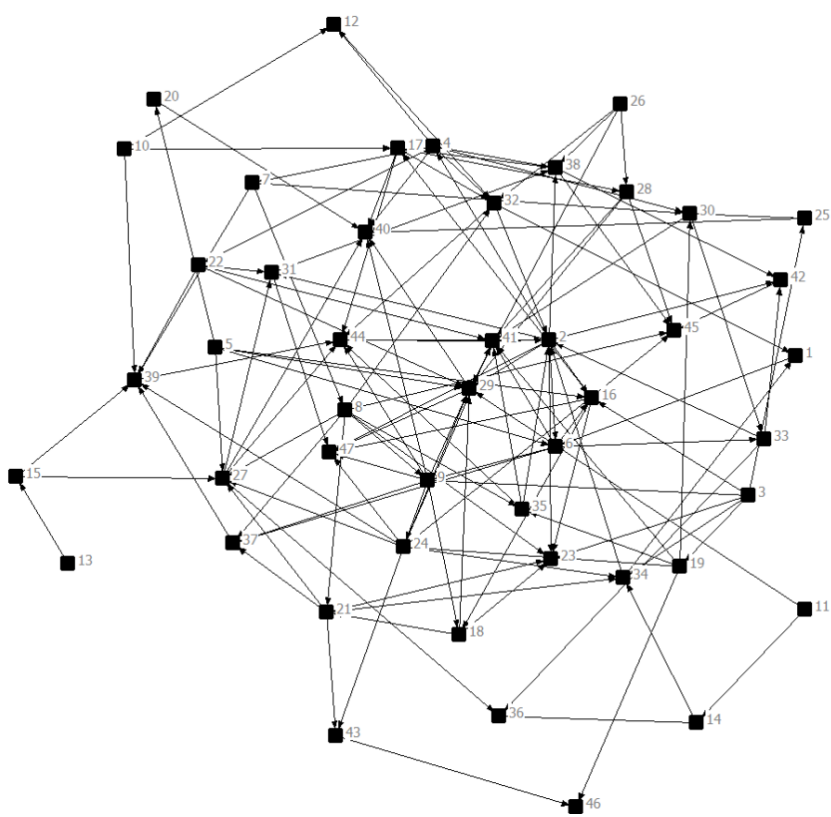

Figura 12. Red de relaciones entre los actores involucrados con el turismo naturista en Cap d'Agde (fuente: elaboración propia con Netdraw)

Si únicamente consideramos las relaciones directas encontradas en Internet entre los 19 clubs swingers de la zona de influencia de Cap d'Agde (los actores del $10 \mathrm{al}$ 28), entonces se obtiene el siguiente sociograma (fig.13), donde podemos comprobar visualmente que existen pocas relaciones directas entre ellos. Hay 7 actores sin ninguna, 11 con una o dos relaciones y 1, el 10 (el club Le Glamour) con tres relaciones (aunque en este caso son relaciones con otros clubs de la misma cadena propietaria del establecimiento).

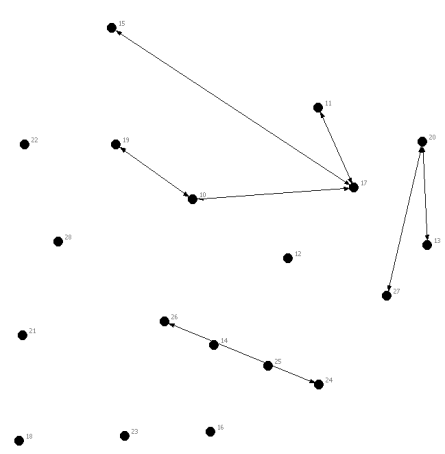

Figura 13. Red de relaciones directas entre los clubs swingers existentes en la zona de influencia de Cap d'Agde (fuente: elaboración propia con Netdraw) 
Si efectuamos el mismo proceso anterior incluyendo también los portales de las comunidades swingers (los actores del 67 al 103), entonces se obtiene el siguiente sociograma (fig.14).

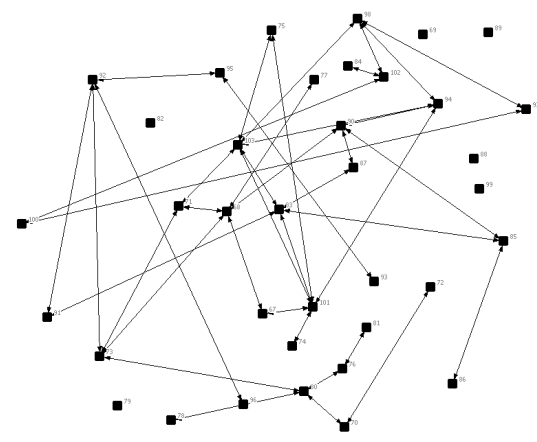

Figura 14. Red de relaciones directas entre los portales de turismo swinger más relacionados con Cap d'Agde (fuente: elaboración propia con Netdraw)

Como se puede observar (fig.14), en este caso el considerable aumento de actores va parejo con la mayor cantidad de relaciones, y solamente hay 6 portales de comunidades swingers sin ninguna relación directa ("links") con el resto de portales, mientras que los restantes portales tienen dos o más conexiones.

Ello demuestra el importante papel de interconectador que dentro de la red realizan los portales de las comunidades swingers.

Además, en el caso del turismo swinger, la proliferación en Internet de "websites" de comunidades swingers, facilitan la interrelación entre los turistas marginales y los clubs swingers, amparada en el anonimato y la facilidad de comunicación; lo que ha servido para construir un extenso entramado de vínculos de acceso a los clubs y demás establecimientos relacionados directamente con esta actividad (hoteles, agencias, resorts, ..) y permitiendo el más rápido desarrollo de este tipo de turismo frente al turismo puramente naturista, más familiar y menos consumista (Prat, 2010).

Así pues, en el caso del turismo swinger de Cap d'Agde, nos encontramos ante una red endogámica de relaciones, cuya dinámica relacional está favorecida desde los portales de las comunidades de parejas swingers.
Figura 15. Red de relaciones directas entre las asociaciones naturistas más relacionadas con Cap d'Agde (fuente: elaboración propia con Netdraw)

Si efectuamos el mismo proceso solamente con las asociaciones naturistas, obtendremos el sociograma siguiente (fig.15), donde podemos ver que entre los 6 nodos existentes existe poca relación, ya que solamente hay "links" entre parejas de asociaciones naturistas.

A continuación, se ha realizado el análisis de las relaciones sociales existentes en los casos de los sociogramas de la fig.11 (red de relaciones entre los actores involucrados con el turismo swinger en Cap d'Agde) y de la fig. 12 (red de relaciones entre los actores involucrados con el turismo naturista en Cap d'Agde), obteniéndose el siguiente cuadro comparativo (fig.16), donde se resumen las características de las relaciones entre los actores para cada una de dichas redes; así como su comparación con los resultados obtenidos anteriormente para el conjunto del turismo marginal de Cap d'Agde, sin distinción de tipologías (fig.10).

Como se puede apreciar en la tabla anterior (fig.16), la dinámica relacional existente en Cap d'Agde para cada uno de estos dos tipos de turismo marginal responde a un modelo distinto, aunque ambos son de baja densidad (en el caso del turismo naturista, la densidad de la red es del $6,34 \%$, mientras que en el caso del turismo swinger es el $4,03 \%$ ), con resultados similares a los del conjunto (es el $7 \%)$.

También podemos observar que en el caso del turismo naturista existe un número pequeño de actores que

\begin{tabular}{|l|c|c|c|}
\hline & $\begin{array}{c}\text { red turismo } \\
\text { naturista }\end{array}$ & $\begin{array}{c}\text { red turismo } \\
\text { swinger }\end{array}$ & $\begin{array}{c}\text { red turismo marginal } \\
\text { conjunto }\end{array}$ \\
\hline $\mathrm{n}^{\circ}$ nodos & 47 & 97 & 103 \\
\hline $\mathrm{n}^{\circ}$ lazos & 137 & 375 & 772 \\
\hline densidad de las relaciones & $0,0634(6,34 \%)$ & $0,0403(4,03 \%)$ & $0,07(7 \%)$ \\
\hline grado de centralización & $20,92 \%$ & $44,98 \%$ & $35,92 \%$ \\
\hline grado de las relaciones & bilaterales & multilaterales & multilaterales \\
\hline $\begin{array}{l}\mathrm{n}^{\circ} \text { actores centrales (con 10 ó } \\
\text { más lazos directos) }\end{array}$ & 4 & 20 & 20 \\
\hline Grado de endogamia & bajo & alto & medio \\
\hline
\end{tabular}

Figura 16. Tabla con las características de las relaciones entre actores (fuente: elaboración propia a partir de Ucinet 6) 
ocupen posiciones centrales en la red (con 10 ó más lazos directos con otros actores) y que, por tanto, impulsen interacciones para resolver conjuntamente actuaciones turísticas, ya que sólo hay 4, con relaciones predominantemente bilaterales; por su parte, el turismo swinger presenta 20 actores centrales, el mismo número que si consideramos el conjunto de ambos turismos, y con unas relaciones predominantemente multilaterales.

Finalmente, el grado de centralización existente en la red del turismo swinger (casi es el 45\%), duplica al existente en la red del turismo naturista (cercano al 21\%), y también es superior al del conjunto del turismo marginal en Cap d'Agde (que es casi del 36\%).

Además, la contrastación de la comparación entre las dinámicas relacionales de ambas redes también nos indica la menor implicación relacional por parte de aquellas entidades más pequeñas, especialmente agencias de viaje, algunos clubs de intercambio y los establecimientos hoteleros ${ }^{16} \mathrm{y}$ de restauración (que son de tamaño mediano y pequeño). Esta débil actividad relacional de una parte de los clubs swingers en Internet, implica un menor uso de los nuevos canales de promoción y comercialización, y de las TIC en general, con las consiguientes mermas de competitividad empresarial, lo que se traduce en una alta rotación de sus propietarios ${ }^{17}$.

Así pues, teniendo en cuenta estas consideraciones, se puede concluir que el turismo swinger en Cap d'Agde (más minoritario que el turismo naturista) posee, sin embargo, una dinámica relacional más intensa, entre los actores involucrados directamente con el barrio naturista, gracias a las "websites" de las comunidades swingers.

En consecuencia, podemos afirmar, en el caso de Cap d'Agde, que la dinámica relacional que generan en un determinado destino las "websites" de las comunidades virtuales relacionadas con el turismo marginal allí presente (en este caso, las comunidades virtuales de swingers y las de los clubs de intercambios), favorecen el desarrollo de este turismo marginal en el destino.

Hipótesis 3: Los potenciales usuarios de estos turismos marginales perciben la utilidad de las opiniones, comentarios y experiencias que otros turistas presentan en las "websites" de dichas comunidades virtuales y, en general, se suelen seguir sus recomendaciones

A partir de los resultados obtenidos de las preguntas de la encuesta relativas a las visitas a algún portal de Internet relacionado con el barrio naturista de Cap d'Agde, a si se habían leído las opiniones y comentarios allí expuestos por otros turistas y a si se había hecho caso de sus recomendaciones, se ha confeccionado el gráfico siguiente (fig.17).

Como se observa en el gráfico anterior (fig.17), respecto a la pregunta de si se había entrado alguna vez en algún portal relacionado con el barrio naturista, el 70\% de las respuestas fueron afirmativas, mientras que el $17 \%$ fueron negativas.

Por su parte, con referencia a la pregunta de si se había leído alguna vez las opiniones y comentarios que escribían en dichos portales otros turistas (y que sólo se reali-

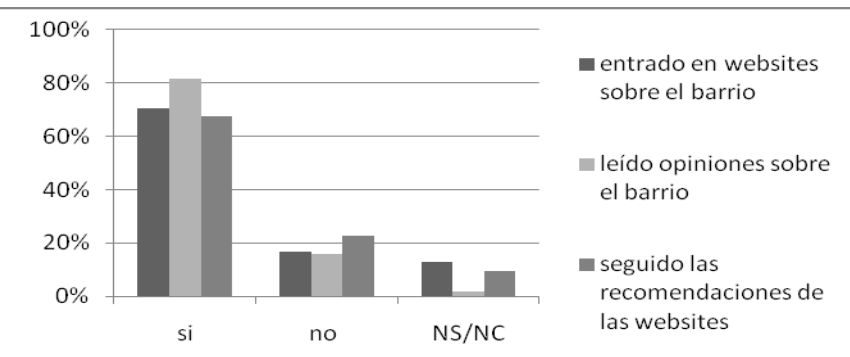

Figura 17. Medio de conocimiento inicial de la existencia del barrio naturista (fuente: elaboración propia partir de la encuesta realizada)

zó a los encuestados que previamente habían respondido afirmativamente a la pregunta anterior), el $82 \%$ de las respuestas fueron positivas, mientras que el $16 \%$ fueron negativas.

Finalmente, en relación a la pregunta de si se habían seguido alguna vez las recomendaciones allí indicadas (que sólo se realizó a los encuestados que previamente habían respondido afirmativamente a la pregunta anterior), el $68 \%$ dijo que sí, mientras que el 10\% afirmó que no lo había hecho nunca.

Así pues, según se desprende de los resultados de la encuesta, los usuarios del turismo marginal de Cap d'Agde, sean naturistas o swingers, tienen conciencia de la utilidad de los portales de Internet relacionados con el barrio naturista, ya que el $70,4 \%$ del total de encuestados afirmó haber leído las recomendaciones entradas en Internet.

Además, con un porcentaje significativo de ellos (el $38,9 \%$ del total de encuestados) han seguido alguna vez las recomendaciones de otros turistas.

En consecuencia, en el caso del barrio naturista de Cap d'Agde, siguiendo la tendencia general de toda la población internauta de Francia (Lacoste, 2010), existe una gran percepción respecto a la utilidad de los portales existentes en Internet sobre el barrio naturista, así como de las opiniones y sugerencias allí expresadas por otros turistas que han visitado dicho lugar.

Por ello, podemos concluir que la utilidad percibida por parte de los potenciales usuarios de los turismo marginales es muy favorable, considerando que la utilidad es el único sentimiento que recurrentemente ha demostrado ser adecuado en entornos tecnológicos para determinar el desarrollo de sentimientos afectivos (Liao et al., 2007) y la intención de uso futuro (Davis et al., 1989; McKnight et al., 2002).

\section{Conclusiones}

Como se ha visto en este artículo con el caso del turismo marginal en el barrio naturista de Cap d'Agde, la dinamización de la actividad turística es una estrategia de desarrollo de muchos destinos; muchas veces de forma planificada por los estamentos públicos, por considerarla necesaria para el desarrollo de la economía local, aprovechando las nuevas demandas de los turistas. 
Paralelamente, las tecnologías de la información (TIC's) han revolucionado la difusión, rápida y extensa, de la información por todo el mundo, y el sector del turismo no ha estado ajeno a este fenómeno, de manera que, utilizando la red de Internet como plataforma de intercambio de información, muchos destinos turísticos se han beneficiado de su uso masivo, gracias a la creación de gran cantidad de "websites" en Internet, con "links" entre ellas, que han generado una compleja red de relaciones, tanto entre los diferentes actores presentes en el destino como entre los turistas que tiene interés en recabar, previamente a su desplazamiento, algún tipo de información sobre dicho destino y el turismo allí ofertado.

En este artículo se ha confirmado que las relaciones sociales generadas entre las "websites" de Internet favorecen el desarrollo del turismo marginal en los destinos.

Además, la dinámica relacional que así se genera entre los actores es más intensa cuantas más potentes (más relacionadas socialmente) sean las comunidades virtuales existentes, relativas a este turismo, de modo dichas comunidades adquieren una gran importancia en la creación de redes sociales complejas, siendo esta importancia mayor cuanto mayor sea su marginalidad, como ocurre en el caso del turismo swinger; de forma que cuanto más se utilicen las nuevas tecnologías (TIC's) para su difusión, más se desarrollan estos turismos en los destinos.

A su vez, los potenciales usuarios de estos turismos marginales perciben la utilidad y suelen seguir en gran medida las recomendaciones que otros turistas escriben en estos portales, ya que cada vez con mayor frecuencia, los viajeros acuden a redes sociales con el fin de obtener información en la que basar sus decisiones de compra de destinos turísticos, y en este sentido, la actitud del viajero hacia la información obtenida en una red social se ha revelado como un factor clave a la hora de explicar este proceso. La confianza en la red social y la utilidad percibida del consejo recibido son dos antecedentes necesarios en la formación de una actitud positiva hacia la información obtenida en la red y la intención del viajero de seguir el consejo obtenido. Esto se debe a que, a la hora de realizar una decisión de compra, los consumidores en general prefieren confiar en fuentes de comunicación personales e informales, ya que, ante la falta de experiencia de primera mano, los consumidores basan sus intenciones de comportamiento en parte de acuerdo con la experiencia de otros consumidores, al considerar que la información proporcionada por individuos similares se va a acercar a su futura experiencia propia, considerándola por tanto información útil para formar sus expectativas.

Por todo ello, como que la participación en redes sociales puede influir el comportamiento del consumidor en el sector turístico, si las asociaciones y entidades que favorecen un tipo concreto de turismo marginal no se implican en su divulgación a través de las relaciones sociales creadas en Internet, entonces este turismo, que, en principio, puede estar cómodamente instalado en la actual situación desde hace años, como puede ser el caso del turismo naturista, puede ver como sus destinos habituales son "cani- balizados" por otras tipologías turísticas más marginales, sostenidas por comunidades virtuales mucho más "agresivas", como ocurre con las comunidades de swingers, que crean unas redes con una dinámica relacional muy intensa y unas relaciones entre "websites" que se mantienen a lo largo del tiempo y que están bien articuladas, no demasiado formalmente, pero que son esenciales en la gestión activa de este turismo en el destino, tanto más al tratarse de un tipo de turismo más minoritario.

En definitiva, en la actualidad, con el uso masivo de las TIC's, disponer de una buena red de conexiones entre "websites" de Internet, es fundamental para la promoción y el desarrollo de los turismo marginales, ya que las "links" entre los distintos portales establecen unas intensas relaciones sociales que ayudan al desarrollo de turismos marginales en los destinos.

\section{Bibliografía}

Allen, J., Massey, D., y Cochrane, A.

1998 Rethinking the region. London: Routledge.

Ávila, R., y Barrado, D.A.

2005 "Nuevas tendencias en el desarrollo de destinos turísticos: marcos conceptuales y operativos para su planificación y gestión”. Cuadernos de Turismo, 15: 27 43.

Bergstrand, C. y Blevins, J.

2000 "Today's Alternative Marriage Styles: The Case of Swingers". Electronic Journal of Human Sexuality, 3: $1-5$.

Borgatti, S.P.

2002 NetDraw: Graph Visualization Software. Harvard: Analytic Technologies.

Borgatti, S.P., Everett, M.G., y Freeman, L.C.

2002: Ucinet 6 forWindows. Software for Social Network Analysis. Harvard: Analytic Technologies.

Brenner, L. y Fricke, J.

2007 "The Evolution of Backpacker destinations: the Case of Zipolite, Mexico". International Journal of Tourism Research, 9: 217-230.

Butler, R.W.

1999 "Sustainable tourism: a state-of-the art review". Tourism Geographies, 1: 7-25.

Chadwick, M.B

2005 "Travel Advocacy: Chadwick Martin Bailey Quantifies the Financial Power of Advocacy in the Travel and Hospitality Industry" (http://www.cmbinfo.com/html/ traveladvocacy.htm)

Cooper, D

2010 "Theorising Nudist Equalirty: An Encounter Between Political Fantasy and Public Appearance". Antipode, 0(00): 1-32.

D'Orlando, F.

2010 "Swinger economics". Social Science Research Network.Working Paper, 1: 1-24.

Davis, F.D., Bagozzi, R.P., y Warshaw, P.R.

1989 "User Acceptance of Computer Technology: A Comparison of Two Theoretical Models". Management Sci- 
ence, 35 (8): 982-1003.

Erickson, B.H., Nosanchuck, T.A, y Lee, E.

1981 "Network Sampling in Practice: Some Second Steps". Social Networks, 2(3): 127-136.

Erickson, B.H. y Nosanchuck, T.A.

1983 “Applied Network Sampling”. Social Networks, 5: 367-382.

Frank, O. y Snidjers, T.

1994 "Estimating the Size of Hidden Populations Using Snowball Sampling". Journal of Official Statistics, 10(1): 53-67.

Frey, R., Malepeyre, L. y Renault, G.

2001 Cap d'Agde 1970-2000, l'histoire de la plus grande station touristique française. Le Cap d'Agde: Éditions Georges Renault.

García Muñoz, T.

1991 "El papel de la Investigación-Acción en el aula: La formación continua del docente y la mejora cualitativa del proceso de enseñanza-aprendizaje”. Caudal 3: 1823.

Granovetter, M.

1976 "Network sampling: some first steps". American Journal of Sociology, 83: 727-729.

Grossetti, M.

2009 "¿Qué es una relacion social ? Un conjunto de mediaciones diádicas”. REDES- Revista hispana para el análisis de redes sociales, 6(2): 44-62.

Harp, S.L.

2011 "The Nacked City of Cap d'Agde: European Nudism and Tourism in Postwar France". En: Zuelow, E.G.E., Touring Beyond the Nation: a transnational approach to european tourism history. Farnham: Ashagate: 3758.

INSEE

2011 Datos estadísticos. Paris: Institut Nacional de la Statistique des Études Économiques.

INTERNET WORLD STATISTIQUES

2010 France (www.internetworldstats.com)

Lacoste, C.

2010 Internet en chiffres en France. Paris: Marques et Tongs.

Liao, C., Chen, J.L. y Yen, D.C.

2007 "Theory of planning behavior (TPB) and customer satisfaction in the continued use of e-service: An integrated model". Computers in Human Behavior, 23(6): 2804-2822.

McKnight, D.H., Choudhury, V. y Kacmar, C.

2002 "The impact of initial consumer trust on intentions to transact with a web site: a trust building mode". Journal of Strategic Information Systems, 11: 297-323.

Merinero, R, y Pulido, J.I.

2009 "Desarrollo turístico y dinámica relacional. Metodología de análisis para la gestión activa de destinos turísticos". Cuadernos de Turismo, 23: 173-193.

Molina, J.L.

2001 El Anàlisis de Redes Sociales: una Introducción. Barcelona: Edicions Bellaterra.
Molina, J.L.

2004. La ciencia de las redes. Apuntes de Ciencia y Tecnología, 11: 36-42.

Monterrubio, J.C.

2008

"Comunidades receptoras y percepciones: un estudio sobre turismo y sexualidad". Teoría y Praxis, 5: 145160

Norrild, J.

2007 "Relación entre turismo, género y sexo. El caso de Buzios-Brasil". Pasos. Revista de Turismo y Patrimonio Cultural: 5(3): 331-341.

Oliver, X. y Alloza, A. Atrapados por el consumo. Barcelona: Vertical.

O’Neill, N. y O’Neill, G. 1972 Open Marriage, A New Lifestyle for Couples. New York: M. Evans \& Company, Inc.

Paasi, A.

2002 "Bounded spaces in the mobile world: Deconstructing 'regional identity", Journal of Economic and Social Geography, 93(2): 137-148.

Pérez, E., Rodríguez, M.E., y Rubio, F. 2003 Turismo en la sociedad de la información: modelos emergentes. El entorno general. Barcelona: UOC. (<http://www.uoc.edu/dt/20352/index.html >)

Prat, J.M.

2010 "Turismo naturista: una apuesta por el turismo marginal. El caso del barrio naturista de Cap d'Agde en Francia”. Cuadernos de Turismo, 26: 207-222.

Priestley, G., y Llurdés, J.C. 2007 "Reflexión final". En: Estrategia y gestión del municipio. Bellaterra: UAB. Escola Universitària de Turisme i Direcció Hotelera: 199-202.

Sanz, L.

2003 "Análisis de Redes Sociales: o como representar las estructuras sociales subyacentes". Apuntes de Ciencia y Tecnología: 7: 21-29.

Suvantola, J.

2002 Tourist Experience of Place. Aldershot: Ashgate.

Townsend, J.G.

2002 "Feminismo, geógrafos y geógrafas feministas y el resurgimiento de la geografía crítica". Documents d'Anàlisi Geogràfica: 40: 175-187.

\section{Notas}

1 Este artículo se desarrolla en el marco del proyecto de investigación "Turismo, territorio e identidad: procesos de revalorización de espacios y actividades en el medio rural español. Un análisis comparado de los casos de Cataluña, Galicia y Murcia”, del Plan Nacional de $\mathrm{I}+\mathrm{D}+\mathrm{i}$, financiado por el Ministerio de Ciencia e Innovación. Referencia: CSO2009-11793

2 Butler estableció su modelo del ciclo de vida de los destinos turísticos (CVDT) basándose en el desarrollo de la vida limitada de los productos y analizando la evolución de la cantidad de turistas recibidos a lo largo del tiempo por un destino (nota del autor) 
3 En este sentido, un estudio de Chadwick Martin Bailey (2005) predijo que, para el año 2006, las recomendaciones y opiniones de otros consumidores podían influenciar de forma directa en 30 billones de dólares las ventas en el sector turístico y, de forma indirecta, en más de 250 billones

4 La denominación "ciencia de las redes" se debe a la incorporación de físicos a un campo conocido hasta ahora como "análisis de redes sociales" (ARS en castellano o SNA, Social Network Analysis en inglés), siendo una red el conjunto de relaciones (líneas, vínculos o lazos) existentes entre una serie definida de elementos (nodos) (Molina, 2004)

5 En este tipo de turismo, además de las parejas swingers, en ocasiones también se añaden personas solas liberales sexualmente pero que no son puramente swingers, ya que ese concepto solo se aplica a las parejas estables (O’Neill y O'Neill, 1972; Bergrstand y Williams, 2000, D’Orlando, 2010)

6 La comuna de Agde ocupa una superfície de 50,8 km2 (INSEE, 2011)

7 Según estimaciones no oficiales, los turistas swingers se gastan anualmente en Cap d'Agde más de 27 millones de euros, y dan trabajo, directa o indirectamente, a más de 1.500 personas (INSEE, 2011)

8 Las exhibiciones sexuales son cada vez más públicas en los atardeceres veraniegos especialmente en la playa y en las dunas, lo que ha derivado en una fuente de constantes conflictos entre ambas comunidades de turistas ((Frey et al., 2001; Monterrubio, 2008; Prat, 2010; Harp, 2011)

9 La encuesta se realizó dentro del trabajo de investigación de miembros del equipo TUDISTAR, cuyos resultados se presentarán en el Pre-Conference Symposium del IGU Comission on Tourism, Leisure and Global Change (Valparaíso, Chile), en noviembre del 2011. Dicho trabajo fue realizado en colaboración con algunas asociaciones locales de Cap d'Agde, especialmente el Movimiento Demócrata del Hérault, entre los meses de septiembre y noviembre de 2010 (nota del autor)

10 Solo se indica la existencia de relación entre los actores, sin establecer el valor de la misma (nota del autor)

11 La encuesta se realizó en el paseo marítimo del puerto de Cap d'Agde, tanto a la población local como a turistas, entre 20 y 60 años de edad, y sin distinción de nacionalidad, sexo ni raza. En total, se realizaron 117 contactos iniciales, que se materializaron en 54 entrevistas personales válidas (el $46,15 \%$ de los contactos iniciales), siendo el $57 \%$ hombres y el $43 \%$ mujeres, ya que el resto se desechó porque no había estado nunca en el barrio naturista. El resultado de la muestra indicó que el $9 \%$ de los encuestados tenía entre 20 y 30 años, el $28 \%$ entre 30 y 40 años, el $44 \%$ entre 40 y 50 años y el 19\% restante entre 50 y 60 años. El 70\% era de nacionalidad francesa y el lugar de residencia del $89 \%$ estaba en Francia (nota del autor)

12 En la comuna de Agde, en el año 2007 había 30.427 segundas residencias (el $70,8 \%$ de las viviendas allí existentes); de las cuales, el $67,4 \%$ se habían construido entre 1975 y 1989, y el $16 \%$ a partir de 1990 (INSEE, 2011)

13 La oferta turística actual de la comuna de Agde se compone de más de 30.000 segundas residencias, 26 campings (con 7.403 emplazamientos disponibles), 25 hoteles (con 678 habitaciones) y 25 hostales y alojamientos de turismo rural (con 678 habitaciones) (INSEE, 2011)

14 Aunque el uso doméstico de Internet empezó en Francia en el año 1994, su despegue definitivo no se produjo hasta la década del 2000 (INTERNET WORLD STATS, 2010)

15 La densidad (D) para matrices simétricas se calcula según la fórmula $\mathrm{D}=\mathrm{r} /(\mathrm{n}(\mathrm{n}-1))$, siendo $\mathrm{r}$ el número de lazos existentes y n el número de nodos (Molina, 2001)

16 El Hotel Eve, de tres estrellas, está situado dentro del barrio nudista de Cap d'Agde y sus 48 habitaciones ofrecen alojamiento para unas 100 personas (nota del autor)

17 Según información facilitada por las asociaciones de empresarios de la comuna de Agde, un 15\% de estos clubs suelen traspasarse al final de cada temporada (nota del autor)
Recibido:
$21 / 02 / 2011$
Reenviado:
$17 / 06 / 2011$
Aceptado:
$10 / 09 / 2011$

Sometido a evaluación por pares anónimos 